\title{
Recursive Model-Based \\ Colour Image Restoration
}

\author{
Michal Haindl \\ Institute of Information Theory and Automation, Academy of Sciences \\ Prague, CZ182 08, Czech Republic \\ haindl@utia.cas.cz
}

\begin{abstract}
This paper presents a derivation of a fast recursive filter for colour image restoration if degradation obeys a linear degradation model with the unknown possibly non-homogeneous point-spread function. Pixels in the vicinity of steep discontinuities are left unrestored to minimize restoration blurring effect. The degraded image is assumed to follow a causal simultaneous multidimensional regressive model and the point-spread function is estimated using the local least-square estimate.
\end{abstract}

\section{Introduction}

Physical imaging systems, the recording medium, the atmosphere are imperfect and thus a recorded image represents a degraded version of the original scene. Similarly an image is usually further corrupted during its processing, transmission or storage. Possible examples are lens defocusing or aberration, noisy transmission channels, motion between camera and scene, etc. The image restoration task is to recover an unobservable image given the observed corrupted image with respect to some statistical criterion. Image restoration is the busy research area for already several decades and many restoration algorithms have been proposed. The simplest restoration method is to smooth the data with an isotropic linear or non-linear shift-invariant low-pass filter. Usual filtering techniques (e.g. median filter, Gaussian low pass filter, band pass filters, etc.) tend to blur the location of boundaries. Several methods [17] try to avoid this problem by using a large number of low-pass filters and combining their outputs. Similarly anisotropic diffusion [18],[5] addresses this problem but it is computationally extremely demanding. Image intensity in this method is allowed to diffuse over time, with the amount of diffusion at a point being inversely proportional to the magnitude of local intensity gradient. A nonlinear filtering method developed by Nitzberg and Shiota [16] uses an offset term to displace kernel centers away from presumed edges and thus to preserve them, however it is not easy to propose all filter parameters to perform satisfactory on variety of different images and the algorithm is very slow. In the exceptional case when the degradation pointspread function is known the Wiener filter [1] or deconvolution methods [12] can be used. Model-based methods use most often Markov random field type of models either in the form of wide sense Markov (regressive models) or strong 
Markov models. The noncausal regressive model used in [3],[4] has the main problem in time consuming iterative solution based on the conjugate gradient method. Similarly Markov random field based restoration methods [7], [6], [13] require time consuming application of Markov chain Monte Carlo methods. Besides this both approaches have solve the problem when to stop these iterative processes. A similar combination of causal and non-causal regressive models as in this paper was used in [14]. However they assume the homogeneous pointspread function and they identify all parameters simultaneously using extremely time consuming iterations of the EM algorithm which is not guaranteed to reach the global optimum. This work generalizes our monospectral restoration method [8] for the multispectral (e.g., colour) images. It is seldom possible to obtain a degradation model analytically from the physics of the problem. More often a limited prior knowledge supports only some elementary assumptions about this process. Usual assumption, accepted also in this work, is that the corruption process can be modeled using a linear degradation model.

\section{Image Model}

Suppose $Y$ represents a true but unobservable colour image defined on finite rectangular $N \times M$ underlying lattice $I$. The observable data are $X$, a version of $Y$ distorted by noise independent of the signal. We assume knowledge of all pixels elements from the reconstructed scene. For the treatment of the more difficult problem when some date are missing see [10], [11]. The image degradation is supposed to be approximated by the linear discrete spatial domain degradation model

$$
X_{r}=\sum_{s \in I_{r}} H_{s} Y_{r-s}+\epsilon_{r}
$$

where $H$ is a discrete representation of the unknown point-spread function and $X_{r}, Y_{r-s}$ corresponding $d \times 1$ multispectral pixels. The point-spread function is assumed to be either homogeneous or it can be non-homogeneous but in this case we assume its slow changes relative to the size of an image. $I_{r}$ is some contextual support set, and a noise vector $\epsilon$ is uncorrelated with the true image, i.e.,

$$
E\{Y \epsilon\}=0
$$

The point-spread function is unknown but such that we can assume the unobservable image $Y$ to be reasonably well approximated by the expectation of the corrupted image

$$
\hat{Y}=E\{X\}
$$

in regions with gradual pixel value changes. The above method (3) changes all pixels in the restored image and thus blurs discontinuities present in the scene although to much less extent than the classical restoration methods due to 
adaptive restoration model (10). This excessive blurring can be avoided if pixels with steep step discontinuities are left unrestored, i.e.,

$$
\hat{Y}_{r}=\left\{\begin{array}{ll}
E\left\{X_{r}\right\} & \text { if }(5) \text { holds } \\
X_{r} & \text { otherwise }
\end{array},\right.
$$

where the adaptive condition (5) is

$$
\left|E\left\{X_{r}\right\}-X_{r}\right|<\frac{1}{n_{s}} \sum_{s}\left|E\left\{X_{r-s}\right\}-X_{r-s}\right| .
$$

The expectation (3) can be expressed as follows:

$$
\begin{aligned}
E\{X\}=\int x p(x) d x= & \int\left(\begin{array}{cccc}
x_{1} & x_{2} & \ldots & x_{M} \\
x_{M+1} & x_{M+2} & \ldots & x_{2 M} \\
\vdots & \vdots & \ddots & \vdots \\
x_{N M-M+1} & x_{N M-M+2} & \ldots & x_{N M}
\end{array}\right) \\
& \prod_{r=1}^{N M} p\left(x_{r} \mid X^{(r-1)}\right) d x_{1} \ldots d x_{N M}
\end{aligned}
$$

where $X^{(r-1)}=\left\{X_{r-1}, \ldots, X_{1}\right\}$ is a set of noisy pixels in some chosen but fixed ordering. For single matrix elements in (6) it holds

$$
\begin{aligned}
E\left\{X_{j}\right\} & =\int x_{j} \prod_{r=1}^{N M} p\left(x_{r} \mid x^{(r-1)}\right) d x_{1} \ldots d x_{N M} \\
& =\int X_{j} \prod_{r=1}^{j} p\left(X_{r} \mid X^{(r-1)}\right) d X_{1} \ldots d X_{j} \\
& =\int E\left\{X_{j} \mid X^{(j-1)}\right\} \prod_{r=1}^{j-1} p\left(X_{r} \mid X^{(r-1)}\right) d X_{1} \ldots d X_{j-1} \\
& =E_{X^{(j-1)}}\left\{E_{X_{j}}\left\{X_{j} \mid X^{(j-1)}\right\}\right\}
\end{aligned}
$$

Let us approximate after having observed $x^{(j-1)}$ the $\hat{Y}_{j}=E\left\{X_{j}\right\}$ by the $E\left\{X_{j} \mid X^{(j-1)}=x^{(j-1)}\right)$ where $x^{(j-1)}$ are known past realization for $j$. Thus we suppose that all other possible realization $x^{(j-1)}$ than the true past pixel values have negligible probabilities. This assumption implies conditional expectations approximately equal to unconditional ones, i.e., then the expectation (7) is

$$
E\left\{X_{j}\right\} \approx E\left\{X_{j} \mid X^{(j-1)}\right\}
$$

and 


$$
\hat{Y}=E\{X\} \approx\left(\begin{array}{ccc}
E\left\{X_{1} \mid x^{(0)}\right\} & \ldots & E\left\{X_{M} \mid x^{(M-1)}\right\} \\
E\left\{X_{M+1} \mid x^{(M)}\right\} & \ldots & E\left\{X_{2 M} \mid x^{(2 M-1)}\right\} \\
\vdots & \ddots & \vdots \\
E\left\{X_{N M-M+1} \mid x^{(N M-M)}\right\} & \ldots & E\left\{X_{N M} \mid x^{(N M-1)}\right\}
\end{array}\right)
$$

Suppose further that the noisy image can be represented by an adaptive causal simultaneous autoregressive model

$$
X_{r}=\sum_{s \in I_{r}^{c}} A_{s} X_{r-s}+\epsilon_{r},
$$

where $\epsilon_{r}$ is a white Gaussian noise vector with zero mean, and a constant but unknown covariance matrix $\Sigma$. The noise vector is uncorrelated with data from a causal neighbourhood $I_{r}^{c}$, but noise vector components can be mutually correlated. The model adaptivity is introduced using the standard exponential forgetting factor technique in parameter learning part of the algorithm. The model can be written in the matrix form

$$
X_{r}=\gamma Z_{r}+\epsilon_{r}
$$

where

$$
\begin{aligned}
\gamma & =\left[A_{1}, \ldots, A_{\eta}\right], \\
\eta & =\operatorname{card}\left(I_{r}^{c}\right)
\end{aligned}
$$

is a $d \times d \eta$ parameter matrix and $Z_{r}$ is a corresponding vector of $X_{r-s}$. To evaluate conditional mean values in (9) the one-step-ahead prediction posterior density $p\left(X_{r} \mid X^{(r-1)}\right)$ is needed. If we assume the normal-Wishart parameter prior for parameters in (10) (alternatively we can assume the Jeffreys parameter prior) this posterior density has the form of d-dimensional Student's probability density

$$
\begin{gathered}
p\left(X_{r} \mid X^{(r-1)}\right)=\frac{\Gamma\left(\frac{\beta(r)-d \eta+d+2}{2}\right)}{\Gamma\left(\frac{\beta(r)-d \eta+2}{2}\right) \pi^{\frac{d}{2}}\left(1+Z_{r}^{T} V_{z(r-1)}^{-1} Z_{r}\right)^{\frac{d}{2}} \lambda_{(r-1)}^{\frac{1}{2}}} \\
\left(1+\frac{\left(X_{r}-\hat{\gamma}_{r-1} Z_{r}\right)^{T} \lambda_{(r-1)}^{-1}\left(X_{r}-\hat{\gamma}_{r-1} Z_{r}\right)}{1+Z_{r}^{T} V_{z(r-1)}^{-1} Z_{r}}\right)^{-\frac{\beta(r)-d \eta+d+2}{2}}
\end{gathered}
$$

with $\beta(r)-d \eta+2$ degrees of freedom, where the following notation is used:

$$
\begin{aligned}
& \beta(r)=\beta(0)+r-1=\beta(r-1)+1, \\
& \beta(0)>1,
\end{aligned}
$$




$$
\begin{aligned}
\hat{\gamma}_{r-1}^{T} & =V_{z(r-1)}^{-1} V_{z x(r-1)} \\
V_{r-1} & =\tilde{V}_{r-1}+I, \\
\tilde{V}_{r-1} & =\left(\begin{array}{cc}
\tilde{V}_{x(r-1)} & \tilde{V}_{z x(r-1)}^{T} \\
\tilde{V}_{z x(r-1)} & \tilde{V}_{z(r-1)}
\end{array}\right), \\
\tilde{V}_{x(r-1)}= & \sum_{k=1}^{r-1} X_{k} X_{k}^{T}, \\
\tilde{V}_{z x(r-1)}= & \sum_{k=1}^{r-1} Z_{k} X_{k}^{T}, \\
\tilde{V}_{z(r-1)}= & \sum_{k=1}^{r-1} Z_{k} Z_{k}^{T}, \\
\lambda_{(r)}= & V_{x(r)}-V_{z x(r)}^{T} V_{z(r)}^{-1} V_{z x(r)} .
\end{aligned}
$$

If $\beta(r-1)>\eta$ then the conditional mean value is

$$
E\left\{X_{r} \mid X^{(r-1)}\right\}=\hat{\gamma}_{r-1} Z_{r}
$$

and it can be efficiently computed using the following recursion

$$
\hat{\gamma}_{r}^{T}=\hat{\gamma}_{r-1}^{T}+\left(1+Z_{r}^{T} V_{z(r-1)}^{-1} Z_{r}\right)^{-1} V_{z(r-1)}^{-1} Z_{r}\left(X_{r}-\hat{\gamma}_{r-1} Z_{r}\right)^{T}
$$

\section{Optimal Contextual Support}

The selection of an appropriate model support $\left(I_{r}^{c}\right)$ is important to obtain good restoration results. If the contextual neighbourhood is too small it can not capture all details of the random field. Inclusion of the unnecessary neighbours on the other hand add to the computational burden and can potentially degrade the performance of the model as an additional source of noise. The optimal Bayesian decision rule for minimizing the average probability of decision error chooses the maximum posterior probability model, i.e., a model $M_{i}$ corresponding to $\max _{j}\left\{p\left(M_{j} \mid X^{(r-1)}\right)\right\}$. If we assume uniform prior for all tested support sets (models) the solution can be found analytically. The most probable model given past data is the model $M_{i}\left(I_{r, i}^{c}\right)$ for which $i=\arg \max _{j}\left\{D_{j}\right\}$.

$$
\begin{aligned}
D_{j}= & -\frac{d}{2} \ln \left|V_{z(r-1)}\right|-\frac{\beta(r)-d \eta+d+1}{2} \ln \left|\lambda_{(r-1)}\right|+\frac{d^{2} \eta}{2} \ln \pi \\
& \sum_{i=1}^{d}\left[\ln \Gamma\left(\frac{\beta(r)-d \eta+d+2-i}{2}\right)-\ln \Gamma\left(\frac{\beta(0)-d \eta+d+2-i}{2}\right)\right] .
\end{aligned}
$$

\section{Global Estimation of the Point-Spread Function}

Similarly with (11) the degradation model (1) can be expressed in the matrix form 


$$
X_{r}=\psi W_{r}+\epsilon_{r}
$$

where $\nu=\operatorname{card}\left(I_{r}\right)$,

$$
\psi=\left[H_{1}, \ldots, H_{\nu}\right],
$$

and $W_{r}$ is a corresponding vector of $Y_{r-s}$. The unobservable $\nu \times 1$ image data vector $W_{r}$ is approximated using (3), (8),(22), i.e.,

$$
\hat{W}_{r}=\left[\hat{\gamma}_{r-s-1} Z_{r}\right]_{s \in I_{r}}^{T} .
$$

In contrast to the model (10) the degradation model (1) is non-causal and hence it has no simple analytical Bayesian parameter estimate. Instead we use the least square estimate

$$
\hat{\psi}=\min _{\psi}\left\{\sum_{\forall r \in I}\left(X_{r}-\psi_{r} \hat{W}_{r}\right)^{T}\left(X_{r}-\psi_{r} \hat{W}_{r}\right)\right\} .
$$

The optimal estimate is $\hat{\psi}^{T}=V_{\hat{W}}^{-1} V_{\hat{W} X}$ where the data gathering matrices $V_{\hat{W}}, V_{\hat{W} X}$ are corresponding analogies with the matrices (18),(19).

\section{Local Estimation of the Point-Spread Function}

If we assume a non-homogeneous slowly changing point-spread function, we can estimate its local value using the local least square estimate

$$
\hat{\psi}_{r}=\min _{\psi_{r}}\left\{\sum_{\forall r \in J_{r}}\left(X_{r}-\psi_{r} \hat{W}_{r}\right)^{T}\left(X_{r}-\psi_{r} \hat{W}_{r}\right)\right\} .
$$

The locally optimal estimate is $\hat{\psi}_{r}^{T}=\tilde{V}_{\hat{W}}^{-1} \tilde{V}_{\hat{W} X}$. The matrices $\tilde{V}_{\hat{W}}, \tilde{V}_{\hat{W} X}$ are computed from subwindows $J_{r} \subset I$. This estimator can be efficiently evaluated using the fast recursive square-root filter introduced in [9].

Table 1. Comparison of the presented method and median filter restoration results for different noise levels

\begin{tabular}{|c|c|c|c|c|c|c|c|c|}
\hline & \multicolumn{6}{|c|}{ Cymbidium image - Gaussian noise } \\
SNR $[d B]$ & 66.5 & 27 & 24.5 & 17.5 & 15.8 & 13.3 & 9.1 & 7.8 \\
$\sigma^{2}$ & 0.001 & 9 & 16 & 81 & 121 & 225 & 625 & 900 \\
MAD - AR & 1.7 & 3.2 & 3.4 & 5.2 & 5.6 & 5.9 & 6.7 & 9.5 \\
$S N R_{i m p}$ & 3.3 & -1.4 & -0.6 & 1.7 & 2.8 & 6.1 & 8.4 & 7.5 \\
MAD - median & 3.1 & 3.9 & 4.1 & 5.3 & 5.9 & 6.0 & 8.8 & 10.3 \\
$S N R_{i m p}$ & -7.8 & -3.3 & -0.7 & 1.6 & 2.6 & 6 & 6.6 & 7.3 \\
\hline
\end{tabular}



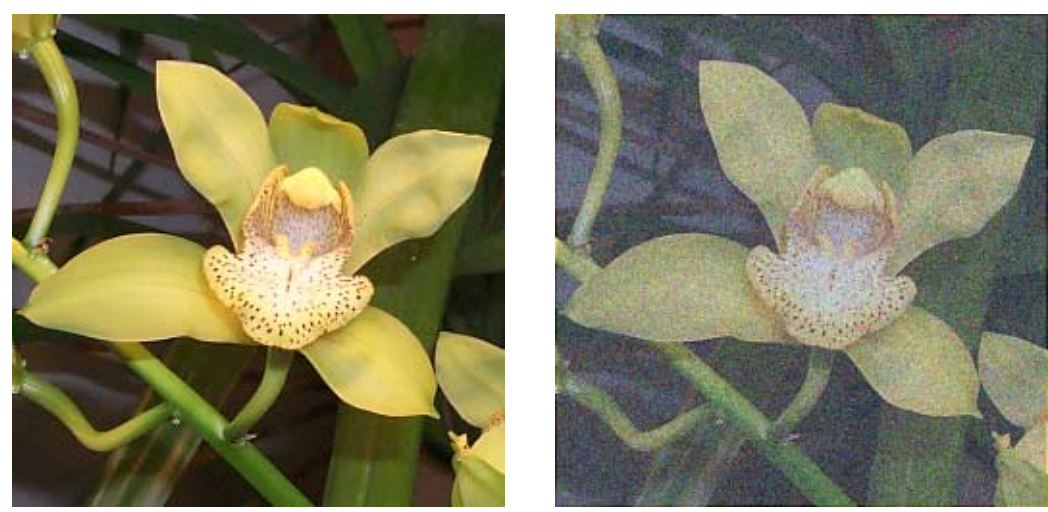

Fig. 1. Original and corrupted ( $\sigma^{2}=900$ white noise) Cymbidium image

\section{Results}

The test image of the Cymbidium orchid (Fig.1-left ), quantized at 256 levels per spectral band, was corrupted by the white Gaussian noise with $\sigma^{2} \in\langle 0.001 ; 900\rangle$ Fig.1-right $\left(\sigma^{2}=900\right)$. The signal-to-noise ratio for these corrupted images is

$$
S N R=10 \log \left(\frac{\operatorname{var}(X)}{\sigma^{2}}\right) \quad d B .
$$

The resulting reconstructed image using our method is on the Fig.2-left $\left(\sigma^{2}=\right.$ 900) while the image Fig.2-right shows reconstruction using identical model but without differentiating discontinuity pixels. Visual comparison of both reconstructed images demonstrates deblurring effect of the presented algorithm.

The performance of the both methods is compared on artificially degraded images (so that the unobservable data are known) using the criterion of mean absolute difference between undegraded and restored pixel values

$$
M A D=\frac{1}{M N d} \sum_{r_{1}=1}^{M} \sum_{r_{2}=1}^{N} \sum_{r_{3}=1}^{d}\left|Y_{r_{1}, r_{2}, r_{3}}-\hat{Y}_{r_{1}, r_{2}, r_{3}}\right|
$$

and the criterion $S N R_{i m p}$ which denotes the improvement in signal-to-noise ratio

$$
S N R_{i m p}=10 \log \left(\frac{\mu(X)}{\mu(\hat{Y})}\right)
$$

where $\mu(X)$ is the mean-square error of $X$.

Both proposed methods are superior over the classical methods using both criteria (30),(31). The edge preserving version of the restoration method demonstrates visible deblurring effect Fig.2-left without significantly affecting numerical complexity of the method. 

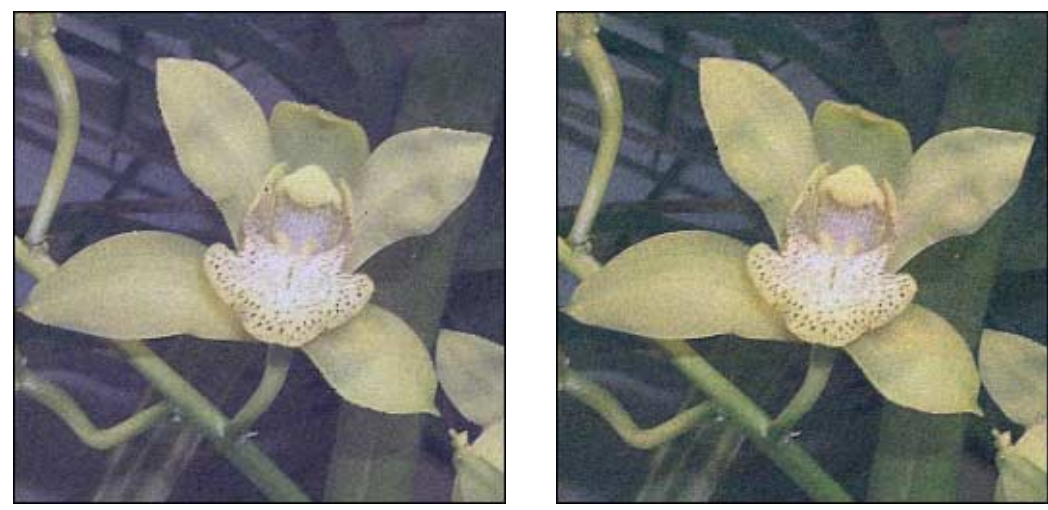

Fig. 2. The reconstructed Cymbidium image using (4),(5) and (3) (right), respectively

The Tab. 1 demonstrates the influence of noise increasing on the performance of our and median filter methods. The proposed method is clearly superior for noisy images.

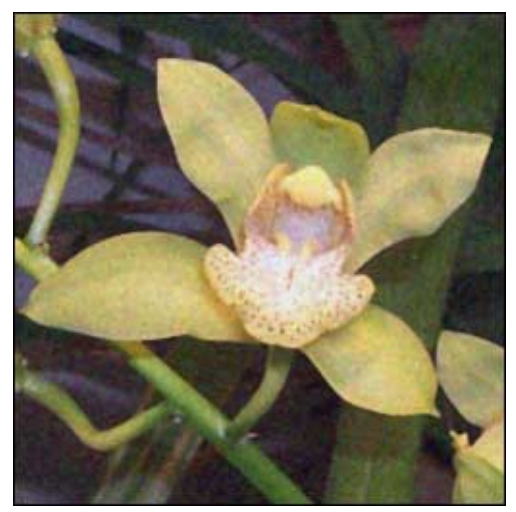

Fig. 3. The reconstructed Cymbidium image using the median filter

\section{Conclusions}

The proposed recursive blur minimizing reconstruction method is very fast (approximately five times faster than the median filter) robust and its reconstruction results surpasses some standard reconstruction methods. Causal models such as 
(10) have obvious advantage to have the analytical solution for parameter estimation, prediction, or model identification tasks. However, this type of models may introduce some artifacts in restored images. These undesirable effects are diminished by introducing adaptivity into the model. This novel formulation allow us to obtain extremely fast adaptive restoration and / or local or global point-spread function estimation which can be easily parallelized. The method can be also easily and naturally generalized for multispectral (e.g. colour, multispectral satellite images) or registered images which is seldom the case for alternative methods. Finally, this method enables to estimate homogeneous or slowly changing non-homogeneous degradation point-spread function.

\section{Acknowledgments}

This research was supported by the GAČR grant no. 102/00/0030 and partially supported by the GAČR grant no. 106/00/1715.

\section{References}

1. Andrews, H. C., Hunt, B.: Digital Image Restoration. Prentice-Hall, Englewood Cliffs (1977) 617

2. Chalmond, B.: Image restoration using an estimated markov model. Signal Processing 15 (1988) 115-129

3. Chellappa, R., Kashyap, R.: Digital image restoration using spatial interaction models. IEEE Trans. Acoustics, Speech and Sig. Proc. 30 (1982) 284-295 618

4. Deguchi, K., Morishita, I.: Two-dimensional auto-regressive model for the representation of random image fields. In: Proc.ICPR Conf., IEEE, Munich (1982) 90-93 618

5. Fischl, B., Schwartz, E.: Learning an integral equation approximation to nonlinear anisotropic diffusion in image processing. IEEE Trans. Pattern Anal. Mach. Int. 19 (1997) 342-352 617

6. Geman, D.: Random fields and inverse problems in imaging. Springer, Berlin, 1990 618

7. Geman, S., Geman, D.: Stochastic relaxation, gibbs distributions and bayesian restoration of images. IEEE Trans. Pattern Anal. Mach. Int. 6 (1984) 721-741 618

8. Haindl, M.: Recursive model-based image restoration. In: Proc. of the 13th ICPR Conf. vol. III, IEEE Press, Barcelona (2000) 346-349 618

9. Haindl, M.: Recursive square-root filters. In: Proc. of the 13th ICPR Conf. vol. II, IEEE Press, Barcelona (2000) 1018-1021 622

10. Haindl, M., Šimberová, S.: A high - resolution radiospectrograph image reconstruction method. Astronomy and Astrophysics, Suppl.Ser. 115 (1996) 189-193 618

11. Haindl, M., Šimberová, S.: A scratch removal method. Kybernetika 34 (1998) 423$428 \quad 618$

12. Hunt, B.: The application of constraint least square estimation to image restoration by digital computer. IEEE Trans. Computers 22 (1973) 805-812 617 
13. Jeffs, B., Pun, W.: Simple shape parameter estimation from blurred observations for a generalized Gaussian mrf image prior used in map restoration. In: Proc. IEEE CVPR Conf., IEEE, San Francisco (1996) 465-468 618

14. Lagendijk, R., Biemond, J., Boekee, D.: Identification and restoration of noisy blurred images using the expectation-maximization algorithm. IEEE Trans. on Acoust., Speech, Signal Processing 38 (1990) 1180-1191 618

15. Marroquin, J., Poggio, T.: Probabilistic solution of ill-posed problems in computational vision. J. Am. Stat. Assoc. 82 (1987) 76-89

16. Nitzberg, M., T. Shiota, T.: Nonlinear image filtering with edge and corner enhancement. IEEE Trans. Pattern Anal. Mach. Int. 16 (1992) 826-833 617

17. Perona, P.: Deformable kernels for early vision. IEEE Trans. Pattern Anal. Mach. Int. 17 (1995) 488-489 617

18. Perona, P., Malik, J.: Scale-space and edge detection using anisotropic diffusion. IEEE Trans. Pattern Anal. Mach. Int. 12 (1990) 629-639 617

19. Reeves, S., Mersereau, R.: Identification by the method of generalized crossvalidation. IEEE Trans. Im. Processing 1 (1992) 301-311 